\title{
Statistical analysis of polycyclic aromatic hydrocarbons concentrations at Baghdad city
}

Received : 7/12/2017

Accepted : 18/1/2018

\author{
Israa M. Almousawi ${ }^{1 *}$, Thikra H. Mathkor ${ }^{1}$, Aessa A. Salih ${ }^{2}$, Adnan H. Afaj $^{2}$, Muthna \\ A.A.Shanshal ${ }^{1}$ \\ ${ }^{1}$ Department of Chemistry, College of Science, University of Baghdad, Baghdad, Iraq, ${ }^{2}$ Ministry of Science and \\ Technology, Baghdad, Iraq \\ *Email: Israa_mousawi@yahoo.com
}

\begin{abstract}
:
The concentrations of 16 polycyclic aromatic hydrocarbons (PAHs) were determined in aerosol samples, which collected for the period (April 2012 to February 2013) at three sites in Baghdad city (AL-Daura refinery, ALDaura power plant and thermal power plant South of Baghdad). These aerosol samples undergone soxhlet extraction.

After the extraction, 138 samples were analyzed by GC-MS to determine sixteen PAH compounds. The average concentrations of individual PAHs were measured in ambient air at the various season. The results indicate the spread of most of the sixteen PAHs at three sites in Baghdad city. Effect of sampling locations, seasons and meteorological conditions on the concentrations of PAHs were studied by SPSS. The positive correlation between the temperature and the total PAH concentration was varied in seasons, which indicated the existence of extrusive relationship between temperature and the total polycyclic aromatic hydrocarbon (TPAH) in the chosen sites.
\end{abstract}

Key words: PAHs in air, toxic PAHs, analysis PAH by GC-MS. 


\section{1-Introduction:}

Polluted air contains one, or more, hazardous substance, pollutant, or contaminant that creates a hazard to general health. The main pollutants found in the air we breathe include, particulate matter, PAHs, lead, ground-level ozone, heavy metals, sulphur dioxide, benzene, carbon monoxide and nitrogen dioxide. Air pollution in cities causes a shorter lifespan for city dwellers [1].

Polycyclic aromatic hydrocarbons (PAHs) are a large group of organic compounds with two or more aromatic rings and are known for their carcinogenic and mutagenic properties and for being responsible of background level contamination on environmental matrices. They are lipophilic and tend to deposit in sediments, soils, and biota [2]. Polycyclic aromatic hydrocarbons (PAHs) originate from the incomplete combustion or pyrolysis of organic matter such as fuel oils [3]. PAHs present in the environment are mainly due to their release from motor vehicles and various industrial sources [4-5] and are widely distributed as pollutants. Recently, some PAHs are suspected to be endocrine disrupters [6-7] and much attention has been focussed on their possible biological effects on human health. Human exposure to PAHs occurs mainly by inhalation of airborne particulates containing PAHs and intake of dietary products contaminated by PAHs [8-9].

PAHs have been thoroughly studied due to their toxicity, persistency and environmental prevalence [10-11]. Such studies are often limited to 16 PAHs, designated as priority pollutants by the United States Environmental Protection Agency (USEPA).

This work presents the results obtained from the determination of the 16 PAHs from samples of the atmospheric air in three locations at Baghdad. Aerosol samples were collected and analyzed by GC-MS device. The calibration curve was used to measure concentrations of PAHs compounds in atmospheric air samples. Statistical analysis was performed using Statistical Package for Social Scientist (SPSS) version 19.

\section{2-Experimental procedure}

\subsection{Description of Sampling sites}

Sampling site selection is an important step in environmental pollution studies. The sampling sites were chosen near the source of emission of pollutants and around it to measure the PAHs concentrations. Three different sites were chosen in Baghdad which included petroleum establishment (AL-Daura refinery) and two power stations (AL-Daura power plant and thermal power plant South of Baghdad). The monitoring of PAHs was focused on the inside of the chosen location and outside location near the urban area surrounding the pollutants source. The selection of these six sites $(1 \rightarrow 6)$ was based on local activities with varying traffic density and industrial activities. It is important to mention, that the selected measurement locations were with the prevailing wind (Northwest - Southeast) as shown in figure 1 .

\subsection{Chemical and Standard material}

Methanol (Fluka 99.8\%) and dichloromethane (Fluka 99.8\%) were used for extraction of $\mathrm{PAH}$ from total suspended particles (TSP). The standard individual material polycyclic aromatic hydrocarbons (PAH) were Naphthalene, Acenaphthylene, Acenaphthene, Fluorene, Phen anthr- ene, Anthracene ,Fluoranthene, Pyrene, Benz(a)anthracene,Chrysene, Benzo(b)fluoranthene ,Benzo (k)fluoranthene, Benzo(a)pyrene, Dibenz- (a,h)anthracene, Benzo(ghi)perylene and Indeno(1,2,3cd)pyrene from (company "Dr. Ehrenstorfer", Germany), purity (99.9\%). The solution concentration of standard individual 
compounds PAHs was ranged (5-350) $\mu \mathrm{g} / \mathrm{l}$. Solutions of these compounds (mixtures or single component) were soluble in acetonitrile or methanol.

\subsection{Standard solution}

The retention time of the individual compounds (PAHs) were measured with range concentration (5-350) $\mu \mathrm{g} / \mathrm{l}$. For the measurements, a 4-points calibration range is used for all PAH. The concentrations of compounds in the calibration curve were used as follow: (3.33, 36.47, 67.41, and 334.22) $\mu \mathrm{g} / \mathrm{L}$.

The target compounds PAHs were identified by comparing with retention time of standard compound and with the mass spectra to estimated molecular weight at the Technology University Darmstadt (TUD) in Germany. The final concentration of compounds PAHs were calculated from calibration curve and converted to $\mu \mathrm{g} / \mathrm{m}^{3}$ [12].

\subsection{Sampling and sample preparation}

Cellulose filters $(4.5 \mathrm{~cm}$ diameter, $47 \mathrm{~mm}$ thickness from Schleicher \& Schuell, Germany) were used to collect aerosol samples from environments. These filters were preheated at $70{ }^{\circ} \mathrm{C}$ for $45 \mathrm{~min}$ prior to use to reduce their water content. Before and after sampling the cellulose filter were weighted on an electronic balance to determine the total suspended particles (TSP) and stored at low temperature in proper container until further extraction and analysis [13].

138 Samples were collected from April 2012 to February of 2013 in sampling sites using a low volume air sampler as called sniffer (RADECO, Inc., England) at a flow rate of $9 \mathrm{f}^{3} \mathrm{~min}^{-1}$, leading to an average sample volume of $7.5 \mathrm{~m}^{3}$. Flow rates were measured at the beginning and at the end of each sampling event. Sampling was done inside and outside of site twice a month for a period of 1.5 hours in the morning and evening using sniffer device. The samples were collected applying (2.0 - 4.0) meter above the ground. The ambient temperature and relative humidity (VAISALA model HM1, Finland), as well as wind rate were measured during the period.

\subsection{Soxhlet extraction}

138 samples were extracted for determining polycyclic aromatic hydrocarbon concentrations in air samples which were collected from sampling sites. Air samples were extracted by soxhlet device [14] using mixture of methanol and dichloromethane (1:1). The soxhlet extractor consisted of a 250 $\mathrm{ml}$ round-bottom flask, $50 \mathrm{ml}$ extractor and water cooled condenser with water bath an arrangement enabling extraction of only one filter sample at time [15].

After extraction, the filter was removed and rinsed with a mixture of methanol and dichloromethane. The filtrate was concentrated to dryness by rotary evaporator (Yamato RE 510) at $60{ }^{\circ} \mathrm{C}$ under gentle vacuum. Then dissolve the dried sample in $5 \mathrm{ml}$ methanol and filtered the solution with micro filter $(0.22 \mu \mathrm{m}$, Minisart, Sartorius Stedim Biotech GmbH 37070 Goettingen, Germany) put in glass container and kept in low temperature.

\subsection{GC/ MS analysis}

All sixteen PAHs were identified and quantified by using a Trace GC/MS spectrometer with MSD, ChemStation software (Agilent Technologies 5975C Series GC/ MSD, USA). Auto-sampler with Triple Axis Detector. Gas chromatography (GC) equipped with a flame ionization detector (FID).

The gas chromatographic column used was Zebron Guardian, phase: ZB-5MS, a nonpolar, packed wih 5\% phenyl methylsilox. Capillary column $(\mathrm{L} 30 \mathrm{~m} \times \mathrm{I}$.D. $0.25 \mathrm{~mm} \times \mathrm{df}=$ $0.25 \mu \mathrm{m})$. High-purity helium $(99.99 \%)$ was used as carrier gas at a flow rate of $1.0 \mathrm{ml} \mathrm{min}$ 
1. The temperatures of the MSD ion source and quadrupole analyzer were kept at $230^{\circ} \mathrm{C}$ and $150{ }^{\circ} \mathrm{C}$, respectively. The detection limit was 1 nanogram/liter. The injected volume was $10 \mu \mathrm{l}$ in splitless mode [16].

The GC oven temperature program was from $75{ }^{\circ} \mathrm{C}$ (hold for $3 \mathrm{~min}$ ) to $235{ }^{\circ} \mathrm{C}$ (20 $\left.{ }^{\circ} \mathrm{C} / \mathrm{min}\right)$, then ramped to $300{ }^{\circ} \mathrm{C}\left(5{ }^{\circ} \mathrm{C} / \mathrm{min}\right)$ and final temperature was $320{ }^{\circ} \mathrm{C}\left(10{ }^{\circ} \mathrm{C} / \mathrm{min}\right.$, hold for $9 \mathrm{~min}$ ). The analysis time per sample was $43 \mathrm{~min}$.

\subsection{Statistical analysis}

Statistical analysis was performed using Statistical Package for Social Scientist (SPSS) version 19, and Microsoft Excel program. SPSS is a comprehensive system for analyzing data. SPSS can take data from almost any type of file and use them to generate tabulated reports, charts, and plots of distributions and trends, descriptive statistics, and complex statistical analysis.

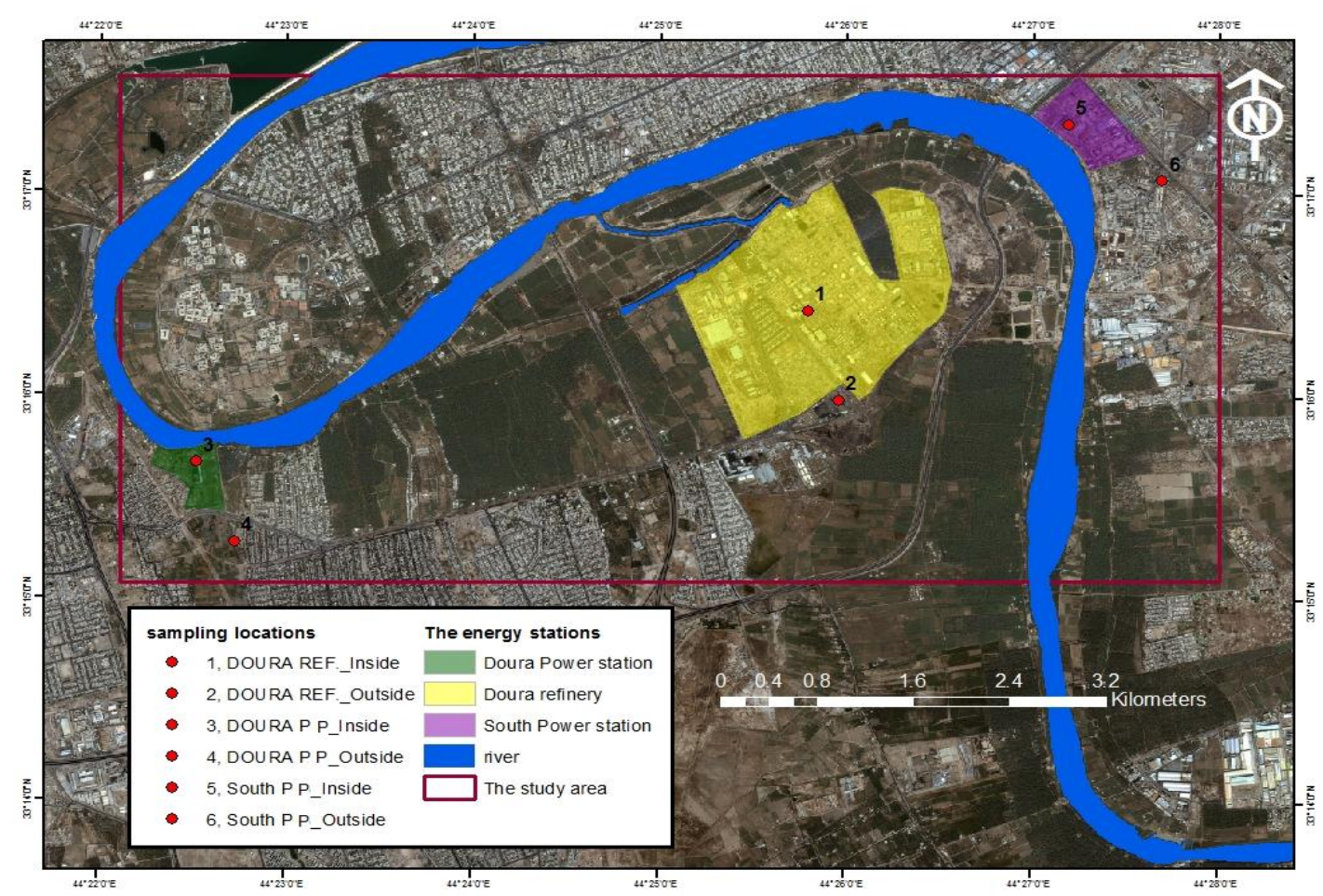

Figure 1: The chosen sites of the energy stations 


\section{Result and discussion}

138 samples were collected and quantified for sixteen PAH compounds by GC/MS device. A single target compound compared with retention time $\left(\mathbf{t}_{\mathbf{R}}\right)$ of individual standard PAHs compounds is given in Table 1.

Both of the concentrations polycyclic aromatic hydrocarbons PAHs and total suspended particles (TSP) were evaluated during the measurement period for selected sites [17-19]. The concentrations of PAHs varied throughout the seasons and in six measuring points at the three sites. The concentrations of TSP measured in different sites show different results. Most concentrations of TSP in different seasons were higher than National Iraqi determinants and international permissible limits [20].

Table 1: Retention time of individual standard PAHs compounds

\begin{tabular}{|c|c|c|c|}
\hline No. & $\begin{array}{c}\text { PAHs / } \\
\text { Abbreviation }\end{array}$ & $\begin{array}{c}\mathbf{t}_{\mathbf{R}} \\
\text { min. }\end{array}$ & $\begin{array}{c}\text { Equation of } \\
\text { a straight } \\
\text { line } \\
\hat{\mathbf{y}}=\mathbf{b c}+\mathbf{a}\end{array}$ \\
\hline 1 & $\begin{array}{l}\text { Naphthalene } \\
\text { (Naph) }\end{array}$ & 8.386 & $\begin{array}{l}264.3 \mathrm{c}+ \\
294.3\end{array}$ \\
\hline 2 & $\begin{array}{l}\text { Acenaphthylene } \\
\text { (Acy ) }\end{array}$ & 10.421 & $\begin{array}{l}276.4 \mathrm{c}- \\
198.8\end{array}$ \\
\hline 3 & $\begin{array}{l}\text { Acenaphthene } \\
\text { (Ace) }\end{array}$ & 10.660 & $\begin{array}{l}191.8 \mathrm{c}- \\
149.3\end{array}$ \\
\hline 4 & Fluorene (Flu ) & 11.301 & $\begin{array}{l}203.7 \mathrm{c}- \\
954.5\end{array}$ \\
\hline 5 & $\begin{array}{l}\text { Phenanthrene } \\
\text { (Phe) }\end{array}$ & 12.622 & $\begin{array}{c}369.6 \mathrm{c}- \\
3367\end{array}$ \\
\hline 6 & Anthracene (Ant) & 12.706 & $\begin{array}{l}381.8 \mathrm{c}- \\
4948\end{array}$ \\
\hline 7 & Fluoranthene (Flt) & 15.181 & $\begin{array}{c}341.5 \mathrm{c}- \\
1559\end{array}$ \\
\hline 8 & Pyrene (Pyr) & 15.900 & $\begin{array}{l}350.5 \mathrm{c}- \\
1400\end{array}$ \\
\hline 9 & $\begin{array}{l}\text { Benzo(a) } \\
\text { Anthracene } \\
(B[a] A)\end{array}$ & 22.549 & $\begin{array}{l}300.1 \mathrm{c}- \\
3164\end{array}$ \\
\hline 10 & Chrysene (Chry) & 22.793 & $\begin{array}{c}306.6 \mathrm{c}- \\
1542\end{array}$ \\
\hline 11 & $\begin{array}{l}\text { Benzo(b) } \\
\text { Fluoranthene } \\
(\mathrm{B}[\mathrm{b}] \mathrm{F})\end{array}$ & 32.452 & $\begin{array}{l}291.7 \mathrm{c}- \\
2297\end{array}$ \\
\hline 12 & $\begin{array}{l}\text { Benzo(k) } \\
\text { Fluoranthene } \\
(\mathrm{B}[\mathrm{k}] \mathrm{F})\end{array}$ & 32.587 & $\begin{array}{l}65.91 \mathrm{c}- \\
274.1\end{array}$ \\
\hline 13 & $\begin{array}{l}\text { Benzo(a) Pyrene } \\
(B[a] P)\end{array}$ & 33.712 & $54.63 \mathrm{c}-39$ \\
\hline 14 & $\begin{array}{l}\text { Indeno }(1,2,3-c d) \\
\text { Pyrene (Ind(cd)P) }\end{array}$ & 37.933 & $\begin{array}{c}107.2 \mathrm{c}+ \\
96.22\end{array}$ \\
\hline 15 & $\begin{array}{l}\text { Dibenzo(a,h) } \\
\text { anthracene (dBA) }\end{array}$ & 38.125 & $\begin{array}{l}147.0 \mathrm{c}- \\
1039\end{array}$ \\
\hline 16 & $\begin{array}{l}\text { Benzo(g,h,i) } \\
\text { Perylene (BghiP) }\end{array}$ & 39.024 & $\begin{array}{c}133.5 \mathrm{c}- \\
534.7\end{array}$ \\
\hline
\end{tabular}




\section{Statistical analysis:}

The concentrations of the individual PAHs and TPAH in the atmosphere resulting from GC/MS analysis were treated using SPSS program in order to study the effects of three factors: sampling locations, seasons and meteorological conditions on the concentrations of PAHs.

\section{$\underline{\text { 3.6.1 Relationship between concentrations of }}$} PAHs and locations:

Analysis of variance (ANOVA- one way) test [21] was applied using Tukey HSD analysis in the SPSS program to evaluate the effects of locations (AL-Daura refinery, Dura power plant and thermal power plant South of Baghdad) on concentrations of PAHs at given season. The results show an increase in the PAHs concentrations significant level ( $\mathrm{p}<$ 0.05 , at $95 \%$ confidence ) in the spring season of most compounds (Naph, Acy, Flu, Phen, Ant, Flt, Pyr, B[a]A and $\mathrm{B}[\mathrm{a}] \mathrm{P}$ ), and insignificant $(p>0.05)$ for variation in the sampling locations of the other seasons.

The change of the compound concentration (increase or decrease), which might be due to the fact that all the sampling points receive their PAHs from one emission source fuel combustion as well as the effects of operating conditions in the establishments and meteorological conditions [22]. Tables (2-4) show some briefed of ANOVA analysis. 
Table 2. Effect of sampling locations on concentrations of PAHs in spring season

\begin{tabular}{|c|c|c|c|c|c|c|c|}
\hline \multirow{2}{*}{$\begin{array}{c}\text { Dependent } \\
\text { Variable }\end{array}$} & \multirow{2}{*}{ Locations } & \multirow{2}{*}{ Locations } & \multirow{2}{*}{$\begin{array}{c}\text { Mean } \\
\text { Difference }\end{array}$} & \multirow{2}{*}{$\begin{array}{l}\text { Std. } \\
\text { Error }\end{array}$} & \multirow{2}{*}{ Sig. } & \multicolumn{2}{|c|}{$\begin{array}{c}\text { 95\% Confidence } \\
\text { Interval }\end{array}$} \\
\hline & & & & & & $\begin{array}{l}\text { Lower } \\
\text { Bound }\end{array}$ & $\begin{array}{l}\text { Upper } \\
\text { Bound }\end{array}$ \\
\hline \multirow[t]{5}{*}{ Naphthalene } & \multirow[t]{2}{*}{ Doura Ref. } & Doura PS & -382.82 & 152.99 & .078 & -810.00 & 44.35 \\
\hline & & South PS & -662.22 & 152.99 & .005 & -1089.40 & -235.05 \\
\hline & Doura PS & Doura Ref. & 382.82 & 152.99 & .078 & -44.35 & 810.00 \\
\hline & \multirow[t]{2}{*}{\begin{tabular}{|l} 
South PS \\
\end{tabular}} & Doura Ref. & 662.22 & 152.99 & .005 & 235.05 & 1089.406 \\
\hline & & Doura PS & 279.40 & 152.99 & .216 & -147.77 & 706.58 \\
\hline \multirow{6}{*}{ Acenaphthylene } & \multirow[t]{2}{*}{ Doura Ref. } & Doura PS & -1.64 & 0.68 & .094 & -3.55 & 0.27 \\
\hline & & South PS & $-3.18^{3}$ & 0.68 & .003 & -5.10 & -1.26 \\
\hline & \multirow[t]{2}{*}{ Doura PS } & Doura Ref. & 1.64 & 0.68 & .094 & -0.27 & 3.55 \\
\hline & & South PS & -1.54 & 0.68 & .115 & -3.46 & 0.37 \\
\hline & \multirow[t]{2}{*}{ South PS } & Doura Ref. & $3.18^{*}$ & 0.68 & .003 & 1.26 & 5.10 \\
\hline & & Doura PS & 1.54 & 0.68 & .115 & -0.37 & 3.46 \\
\hline \multirow[t]{5}{*}{ Fluorene } & \multirow[t]{2}{*}{ Doura Ref. } & Doura PS & -5.41 & 2.80 & .186 & -13.25 & 2.42 \\
\hline & & South PS & $-12.94^{*}$ & 2.80 & .003 & -20.78 & -5.10 \\
\hline & Doura PS & Doura Ref. & 5.41 & 2.80 & .186 & -2.42 & 13.25 \\
\hline & \multirow[t]{2}{*}{\begin{tabular}{|l} 
South PS \\
\end{tabular}} & Doura Ref. & 12.94 & 2.80 & .003 & 5.10 & 20.78 \\
\hline & & Doura PS & 7.52 & 2.80 & .060 & -0.314 & 15.36 \\
\hline \multirow[t]{6}{*}{ Phenanthrene } & \multirow[t]{2}{*}{ Doura Ref. } & Doura PS & -29.24 & 19.182 & .325 & -82.80 & 24.31 \\
\hline & & South PS & $-87.76^{*}$ & 19.182 & .003 & -141.32 & -34.20 \\
\hline & \multirow[t]{2}{*}{\begin{tabular}{|l} 
Doura PS \\
\end{tabular}} & Doura Ref. & 29.24 & 19.182 & .325 & -24.31 & 82.80 \\
\hline & & South PS & $-58.51^{*}$ & 19.182 & .033 & -112.07 & -4.96 \\
\hline & \multirow[t]{2}{*}{\begin{tabular}{|l} 
South PS \\
\end{tabular}} & Doura Ref. & $87.76^{*}$ & 19.182 & .003 & 34.20 & 141.32 \\
\hline & & Doura PS & 58.51 & 19.182 & .033 & 4.96 & 112.07 \\
\hline \multirow[t]{6}{*}{ Anthracene } & \multirow[t]{2}{*}{ Doura Ref. } & Doura PS & -19.46 & 843.65 & 1.00 & -2374.95 & 2336.02 \\
\hline & & South PS & $-3136.99^{*}$ & 843.65 & .012 & -5492.48 & -781.51 \\
\hline & \multirow[t]{2}{*}{\begin{tabular}{|l} 
Doura PS \\
\end{tabular}} & Doura Ref. & 19.46 & 843.65 & 1.00 & -2336.02 & 2374.95 \\
\hline & & South PS & $-3117.53^{*}$ & 843.65 & .012 & -5473.02 & -762.04 \\
\hline & \begin{tabular}{|l|} 
South PS \\
\end{tabular} & Doura Ref. & $3136.99^{*}$ & 843.65 & .012 & 781.51 & 5492.48 \\
\hline & & Doura PS & $3117.53^{*}$ & 843.65 & .012 & 762.04 & 5473.02 \\
\hline Fluoranthene & Doura Ref. & Doura PS & -7.34 & 4.38 & .266 & -19.58 & 4.89 \\
\hline & & South PS & -17.06 & 4.38 & .009 & -29.30 & -4.83 \\
\hline & \begin{tabular}{|l} 
South PS \\
\end{tabular} & Doura Ref. & $17.06^{*}$ & 4.38 & .009 & 4.83 & 29.30 \\
\hline & & Doura PS & 9.72 & 4.38 & .121 & -2.51 & 21.95 \\
\hline Pyrene & Doura Ref. & Doura PS & -15.05 & 9.40 & .294 & -41.31 & 11.20 \\
\hline & & South PS & $-36.64 *$ & 9.40 & .009 & -62.91 & -10.38 \\
\hline & Doura PS & Doura Ref. & 15.05 & 9.40 & .294 & -11.20 & 41.31 \\
\hline & & South PS & -21.59 & 9.40 & .108 & -47.85 & 4.67 \\
\hline & South PS & Doura Ref. & $36.64^{*}$ & 9.40 & .009 & 10.38 & 62.91 \\
\hline Benzo[a]anthracene & Doura Ref. & Doura PS & -2.93 & 0.80 & .014 & -5.18 & -0.68 \\
\hline & & South PS & $-3.20^{3}$ & 0.80 & .008 & -5.46 & -0.95 \\
\hline & Doura PS & Doura Ref. & $2.93^{*}$ & 0.80 & .014 & .68 & 5.18 \\
\hline & South PS & Doura Ref. & 3.20 & 0.80 & .008 & 0.95 & 5.46 \\
\hline
\end{tabular}


Table 3. Effect of sampling locations on concentrations of PAHs in summer season.

\begin{tabular}{|c|c|c|c|c|c|c|c|}
\hline \multirow[t]{2}{*}{$\begin{array}{c}\text { Dependent } \\
\text { Variable }\end{array}$} & \multirow[t]{2}{*}{ Locations } & \multirow[t]{2}{*}{ Locations } & \multirow[t]{2}{*}{$\begin{array}{c}\text { Mean } \\
\text { Difference }\end{array}$} & \multirow[t]{2}{*}{$\begin{array}{l}\text { Std. } \\
\text { Error }\end{array}$} & \multirow[t]{2}{*}{ Sig. } & \multicolumn{2}{|c|}{$\begin{array}{l}\text { 95\% Confidence } \\
\text { Interval }\end{array}$} \\
\hline & & & & & & $\begin{array}{l}\text { Lower } \\
\text { Bound }\end{array}$ & $\begin{array}{l}\text { Upper } \\
\text { Bound }\end{array}$ \\
\hline \multirow[t]{6}{*}{ Naphthalene } & \multirow[t]{2}{*}{ Doura Ref. } & Doura PS & 251.79 & 355.18 & .764 & -739.88 & 1243.47 \\
\hline & & South PS & -8.54 & 355.18 & 1.000 & -1000.21 & 983.13 \\
\hline & \multirow[t]{2}{*}{ Doura PS } & Doura Ref. & -251.79 & 355.18 & .764 & -1243.47 & 739.88 \\
\hline & & \begin{tabular}{|l|} 
South PS \\
\end{tabular} & -260.33 & 355.18 & .751 & -1252.01 & 731.34 \\
\hline & \multirow[t]{2}{*}{ South PS } & Doura Ref. & 8.54 & 355.18 & 1.000 & -983.13 & 1000.21 \\
\hline & & Doura PS & 260.33 & 355.18 & .751 & -731.34 & 1252.01 \\
\hline \multirow[t]{6}{*}{ Acenaphthylene } & \multirow[t]{2}{*}{ Doura Ref. } & Doura PS & -4.91 & 7.91 & .812 & -26.99 & 17.164 \\
\hline & & South PS & -8.05 & 7.91 & .584 & -30.13 & 14.02 \\
\hline & \multirow[t]{2}{*}{ Doura PS } & Doura Ref. & 4.91 & 7.91 & .812 & -17.16 & 26.99 \\
\hline & & \begin{tabular}{|l|} 
South PS \\
\end{tabular} & -3.13 & 7.91 & .918 & -25.21 & 18.94 \\
\hline & \multirow[t]{2}{*}{ South PS } & Doura Ref. & 8.05 & 7.91 & .584 & -14.02 & 30.13 \\
\hline & & Doura PS & 3.13 & 7.91 & .918 & -18.94 & 25.21 \\
\hline \multirow[t]{6}{*}{ Acenaphthene } & \multirow[t]{2}{*}{ Doura Ref. } & Doura PS & -6.51 & 20.56 & .946 & -63.93 & 50.89 \\
\hline & & South PS & -23.81 & 20.56 & .505 & -81.22 & 33.60 \\
\hline & \multirow[t]{2}{*}{ Doura PS } & Doura Ref. & 6.51 & 20.56 & .946 & -50.89 & 63.93 \\
\hline & & South PS & -17.29 & 20.56 & .689 & -74.70 & 40.12 \\
\hline & \multirow[t]{2}{*}{ South PS } & Doura Ref. & 23.81 & 20.56 & .505 & -33.60 & 81.22 \\
\hline & & Doura PS & 17.29 & 20.56 & .689 & -40.12 & 74.70 \\
\hline \multirow[t]{6}{*}{ Fluorene } & \multirow[t]{2}{*}{ Doura Ref. } & Doura PS & -11.74 & 23.58 & .874 & -77.60 & 54.11 \\
\hline & & South PS & -24.46 & 23.58 & .574 & -90.32 & 41.39 \\
\hline & \multirow[t]{2}{*}{ Doura PS } & Doura Ref. & 11.74 & 23.58 & .874 & -54.11 & 77.60 \\
\hline & & South PS & -12.71 & 23.58 & .854 & -78.58 & 53.14 \\
\hline & \multirow[t]{2}{*}{ South PS } & Doura Ref. & 24.46 & 23.58 & .574 & -41.39 & 90.32 \\
\hline & & Doura PS & 12.719 & 23.58 & .854 & -53.14 & 78.58 \\
\hline
\end{tabular}

Std. Error $=$ standard error, Sig. $=$ significant level. 
. Table 4. Effect of sampling locations on concentrations of PAHs in autumn season.

\begin{tabular}{|c|c|c|c|c|c|c|c|}
\hline \multirow{2}{*}{$\begin{array}{c}\text { Dependent } \\
\text { Variable }\end{array}$} & \multirow[b]{2}{*}{ Locations } & \multirow[b]{2}{*}{ Locations } & \multirow{2}{*}{$\begin{array}{c}\text { Mean } \\
\text { Difference }\end{array}$} & \multirow{2}{*}{$\begin{array}{l}\text { Std. } \\
\text { Error }\end{array}$} & \multirow[b]{2}{*}{ Sig. } & \multicolumn{2}{|c|}{$\begin{array}{l}\text { 95\% Confidence } \\
\text { Interval }\end{array}$} \\
\hline & & & & & & $\begin{array}{l}\text { Lower } \\
\text { Bound }\end{array}$ & $\begin{array}{l}\text { Upper } \\
\text { Bound }\end{array}$ \\
\hline \multirow{6}{*}{$\begin{array}{r}\text { Benzo[b] } \\
\text { fluoranthene }\end{array}$} & Doura & Doura PS & -.814 & 4.39 & .981 & -13.08 & 11.46 \\
\hline & Ref. & South PS & -5.72 & 4.39 & .429 & -17.99 & 6.55 \\
\hline & Doura PS & $\begin{array}{l}\text { Doura } \\
\text { Ref. }\end{array}$ & .81 & 4.39 & .981 & -11.46 & 13.08 \\
\hline & & South PS & -4.90 & 4.39 & .528 & -17.18 & 7.36 \\
\hline & South PS & $\begin{array}{r}\text { Doura } \\
\text { Ref. }\end{array}$ & 5.72 & 4.39 & .429 & -6.55 & 17.99 \\
\hline & & Doura PS & 4.91 & 4.39 & .528 & -7.36 & 17.18 \\
\hline \multirow{6}{*}{$\begin{array}{r}\text { Benzo[k] } \\
\text { fluoranthene }\end{array}$} & Doura & Doura PS & -.03 & 4.65 & 1.000 & -13.02 & 12.95 \\
\hline & Ref. & South PS & -5.06 & 4.65 & .545 & -18.05 & 7.93 \\
\hline & Doura PS & $\begin{array}{l}\text { Doura } \\
\text { Ref. }\end{array}$ & 0.03 & 4.65 & 1.000 & -12.95 & 13.02 \\
\hline & & South PS & -5.02 & 4.65 & .549 & -18.01 & 7.96 \\
\hline & South PS & $\begin{array}{r}\text { Doura } \\
\text { Ref. }\end{array}$ & 5.06 & 4.65 & .545 & -7.93 & 18.05 \\
\hline & & Doura PS & 5.03 & 4.65 & .549 & -7.96 & 18.02 \\
\hline \multirow[t]{6}{*}{ Benzo[a]pyrene } & Doura & Doura PS & 1.24 & 2.47 & .871 & -5.66 & 8.15 \\
\hline & Ref. & South PS & -2.28 & 2.47 & .640 & -9.19 & 4.62 \\
\hline & Doura PS & $\begin{array}{l}\text { Doura } \\
\text { Ref. }\end{array}$ & -1.24 & 2.47 & .871 & -8.15 & 5.66 \\
\hline & & South PS & -3.53 & 2.47 & .368 & -10.43 & 3.37 \\
\hline & South PS & $\begin{array}{l}\text { Doura } \\
\text { Ref. }\end{array}$ & 2.28 & 2.47 & .640 & -4.62 & 9.19 \\
\hline & & Doura PS & 3.53 & 2.47 & .368 & -3.37 & 10.43 \\
\hline \multirow{6}{*}{$\begin{array}{r}\text { Indeno[1,2,3- } \\
\text { cd]pyrene }\end{array}$} & Doura & Doura PS & -1.58 & 7.79 & .977 & -23.35 & 20.18 \\
\hline & Ref. & South PS & -10.25 & 7.79 & .422 & -32.03 & 11.51 \\
\hline & Doura PS & $\begin{array}{l}\text { Doura } \\
\text { Ref. }\end{array}$ & 1.58 & 7.79 & .977 & -20.18 & 23.35 \\
\hline & & South PS & -8.66 & 7.79 & .531 & \begin{tabular}{|l|}
-30.43 \\
\end{tabular} & 13.10 \\
\hline & South PS & $\begin{array}{l}\text { Doura } \\
\text { Ref. }\end{array}$ & 10.25 & 7.79 & .422 & -11.51 & 32.02 \\
\hline & & Doura PS & 8.66 & 7.79 & .531 & -13.10 & 30.43 \\
\hline
\end{tabular}

\subsubsection{Relationship between concentrations of PAHs and seasons}

Analysis of variance (ANOVA- one way) test was applied using Tukey HSD analysis to determine the effects of variation seasons on concentrations of PAHs at given location. The results are showing significant level $(\mathrm{p}<0.05$ at
95\% confidence) in the concentration of some compounds (Naph, Flu and Phen) at AL-Daura refinery and significant level in the concentrations of anthracene at thermal power plant South of Baghdad, while insignificant level in the other concentrations of PAHs. Tables (5-6) show some results of ANOVA analysis. 
Table 5. Effect of the seasons on concentrations of PAHs at AL-Daura refinery.

\begin{tabular}{|c|c|c|c|c|c|c|c|}
\hline \multirow{2}{*}{$\begin{array}{c}\text { Dependent } \\
\text { Variable }\end{array}$} & \multirow{2}{*}{ Seasons } & \multirow{2}{*}{ Seasons } & \multirow{2}{*}{$\begin{array}{c}\text { Mean } \\
\text { Difference }\end{array}$} & \multirow{2}{*}{$\begin{array}{l}\text { Std. } \\
\text { Error }\end{array}$} & \multirow{2}{*}{ Sig. } & \multicolumn{2}{|c|}{$\begin{array}{l}\text { 95\% Confidence } \\
\text { Interval }\end{array}$} \\
\hline & & & & & & $\begin{array}{l}\text { Lower } \\
\text { Bound }\end{array}$ & $\begin{array}{l}\text { Upper } \\
\text { Bound }\end{array}$ \\
\hline \multirow[t]{12}{*}{ Naphthalene } & \multirow[t]{3}{*}{ Summer } & Autumn & $434.864^{*}$ & 86.740 & 0.001 & 177.341 & 692.387 \\
\hline & & Winter & $440.948^{*}$ & 86.740 & 0.001 & 183.424 & 698.471 \\
\hline & & Spring & $413.689^{*}$ & 86.740 & 0.002 & 156.166 & 671.213 \\
\hline & \multirow[t]{3}{*}{ Autumn } & Summer & $-434.864^{*}$ & 86.740 & 0.001 & -692.387 & -177.341 \\
\hline & & Winter & 6.083 & 86.740 & 1.00 & -251.439 & 263.6071 \\
\hline & & Spring & -21.174 & 86.740 & 0.995 & -278.698 & 236.348 \\
\hline & \multirow[t]{3}{*}{\begin{tabular}{|l|} 
Winter \\
\end{tabular}} & Summer & $-440.948^{*}$ & 86.740 & 0.001 & -698.471 & -183.424 \\
\hline & & Autumn & -6.083 & 86.740 & 1.00 & -263.607 & 251.439 \\
\hline & & Spring & -27.258 & 86.740 & 0.989 & -284.781 & 230.264 \\
\hline & \multirow[t]{3}{*}{ Spring } & Summer & $-413.689^{*}$ & 86.740 & 0.002 & -671.213 & -156.166 \\
\hline & & \begin{tabular}{|l|} 
Autumn \\
\end{tabular} & 21.1740 & 86.740 & 0.995 & -236.348 & 278.698 \\
\hline & & Winter & 27.2585 & 86.740 & 0.989 & -230.264 & 284.781 \\
\hline \multirow[t]{9}{*}{ Fluorene } & \multirow[t]{3}{*}{ Summer } & Autumn & $3.752^{*}$ & 1.0207 & 0.015 & .721 & 6.782 \\
\hline & & Winter & $3.961^{*}$ & 1.0207 & 0.010 & .9309 & 6.992 \\
\hline & & Spring & 1.077 & 1.0207 & 0.722 & -1.953 & 4.107 \\
\hline & \multirow[t]{3}{*}{\begin{tabular}{|l|} 
Autumn \\
\end{tabular}} & Summer & $-3.752 *$ & 1.0207 & 0.015 & -6.782 & -.721 \\
\hline & & Winter & .209 & 1.0207 & 0.997 & -2.821 & 3.240 \\
\hline & & Spring & -2.675 & 1.0207 & 0.090 & -5.705 & .355 \\
\hline & \multirow[t]{3}{*}{ Winter } & Summer & $-3.961^{*}$ & 1.0207 & 0.010 & -6.992 & -.930 \\
\hline & & \begin{tabular}{|l|} 
Autumn \\
\end{tabular} & -.209 & 1.0207 & 0.997 & -3.240 & 2.821 \\
\hline & & Spring & -2.884 & 1.0207 & 0.064 & -5.915 & .1460 \\
\hline \multirow[t]{12}{*}{ Phenanthrene } & \multirow[t]{3}{*}{ Summer } & Autumn & $20.250 *$ & 6.467 & 0.038 & 1.0475 & 39.452 \\
\hline & & Winter & $20.428^{*}$ & 6.467 & 0.036 & 1.226 & 39.630 \\
\hline & & Spring & 14.690 & 6.467 & 0.160 & -4.512 & 33.892 \\
\hline & \multirow{3}{*}{ Autumn } & Summer & $-20.250^{*}$ & 6.4678 & 0.038 & -39.452 & -1.0475 \\
\hline & & Winter & .1785 & 6.4678 & 1.00 & -19.023 & 19.380 \\
\hline & & Spring & -5.5597 & 6.4678 & 0.825 & -24.762 & 13.642 \\
\hline & \multirow[t]{3}{*}{\begin{tabular}{|l|} 
Winter \\
\end{tabular}} & Summer & $-20.428^{*}$ & 6.4678 & 0.036 & -39.630 & -1.226 \\
\hline & & Autumn & -.1785 & 6.467 & 1.00 & -19.380 & 19.023 \\
\hline & & Spring & -5.7382 & 6.467 & 0.812 & -24.94 & 13.464 \\
\hline & \multirow[t]{3}{*}{ Spring } & Summer & -14.690 & 6.4678 & 0.160 & -33.892 & 4.512 \\
\hline & & Autumn & 5.5597 & 6.4678 & 0.825 & -13.642 & 24.762 \\
\hline & & Winter & 5.7382 & 6.467 & 0.812 & -13.464 & 24.940 \\
\hline
\end{tabular}

* The mean difference is significant at the 0.05 level (95\% confidence). 
Table 6. Effect of season on concentrations of PAHs at thermal South power plant.

\begin{tabular}{|c|c|c|c|c|c|c|c|}
\hline \multirow{2}{*}{$\begin{array}{l}\text { Dependent } \\
\text { Variable }\end{array}$} & \multirow{2}{*}{ Seasons } & \multirow{2}{*}{ Seasons } & \multirow{2}{*}{$\begin{array}{c}\text { Mean } \\
\text { Difference }\end{array}$} & \multirow{2}{*}{$\begin{array}{l}\text { Std. } \\
\text { Error }\end{array}$} & \multirow{2}{*}{ Sig. } & \multicolumn{2}{|c|}{$\begin{array}{l}\text { 95\% Confidence } \\
\text { Interval }\end{array}$} \\
\hline & & & & & & $\begin{array}{l}\text { Lower } \\
\text { Bound }\end{array}$ & $\begin{array}{l}\text { Upper } \\
\text { Bound }\end{array}$ \\
\hline \multirow[t]{12}{*}{ Naphthalene } & \multirow[t]{3}{*}{ Summer } & Autumn & $434.864^{*}$ & 86.740 & 0.001 & 177.341 & 692.387 \\
\hline & & \begin{tabular}{|l|} 
Winter \\
\end{tabular} & $440.948^{*}$ & 86.740 & 0.001 & 183.424 & 698.471 \\
\hline & & Spring & $413.689^{*}$ & 86.740 & 0.002 & 156.166 & 671.213 \\
\hline & \multirow[t]{3}{*}{\begin{tabular}{|l} 
Autumn \\
\end{tabular}} & Summer & $-434.864^{*}$ & 86.740 & 0.001 & -692.387 & -177.341 \\
\hline & & Winter & 6.083 & 86.740 & 1.00 & -251.439 & 263.6071 \\
\hline & & Spring & -21.174 & 86.740 & 0.995 & -278.698 & 236.348 \\
\hline & \multirow[t]{3}{*}{ Winter } & Summer & $-440.948^{*}$ & 86.740 & 0.001 & -698.471 & -183.424 \\
\hline & & Autumn & -6.083 & 86.740 & 1.00 & -263.607 & 251.439 \\
\hline & & Spring & -27.258 & 86.740 & 0.989 & -284.781 & 230.264 \\
\hline & \multirow{3}{*}{\begin{tabular}{|l} 
Spring \\
\end{tabular}} & Summer & $-413.689^{*}$ & 86.740 & 0.002 & -671.213 & -156.166 \\
\hline & & \begin{tabular}{|l} 
Autumn \\
\end{tabular} & 21.1740 & 86.740 & 0.995 & -236.348 & 278.698 \\
\hline & & Winter & 27.2585 & 86.740 & 0.989 & -230.264 & 284.781 \\
\hline \multirow[t]{9}{*}{ Fluorene } & \multirow[t]{3}{*}{ Summer } & Autumn & $3.752^{*}$ & 1.0207 & 0.015 & .721 & 6.782 \\
\hline & & \begin{tabular}{|l} 
Winter \\
\end{tabular} & $3.961^{*}$ & 1.0207 & 0.010 & .9309 & 6.992 \\
\hline & & Spring & 1.077 & 1.0207 & 0.722 & -1.953 & 4.107 \\
\hline & \multirow[t]{3}{*}{ Autumn } & Summer & $-3.752^{*}$ & 1.0207 & 0.015 & -6.782 & -.721 \\
\hline & & Winter & .209 & 1.0207 & 0.997 & -2.821 & 3.240 \\
\hline & & \begin{tabular}{|l} 
Spring \\
\end{tabular} & -2.675 & 1.0207 & 0.090 & -5.705 & .355 \\
\hline & \multirow{3}{*}{ Winter } & Summer & $-3.961^{*}$ & 1.0207 & 0.010 & -6.992 & -.930 \\
\hline & & Autumn & -.209 & 1.0207 & 0.997 & -3.240 & 2.821 \\
\hline & & \begin{tabular}{|l} 
Spring \\
\end{tabular} & -2.884 & 1.0207 & 0.064 & -5.915 & .1460 \\
\hline \multirow[t]{12}{*}{ Phenanthrene } & \multirow[t]{3}{*}{ Summer } & Autumn & $20.250 *$ & 6.467 & 0.038 & 1.0475 & 39.452 \\
\hline & & Winter & $20.428^{*}$ & 6.467 & 0.036 & 1.226 & 39.630 \\
\hline & & \begin{tabular}{|l|} 
Spring \\
\end{tabular} & 14.690 & 6.467 & 0.160 & -4.512 & 33.892 \\
\hline & \multirow[t]{3}{*}{\begin{tabular}{|l} 
Autumn \\
\end{tabular}} & Summer & $-20.250^{*}$ & 6.4678 & 0.038 & -39.452 & -1.0475 \\
\hline & & Winter & .1785 & 6.4678 & 1.00 & -19.023 & 19.380 \\
\hline & & Spring & -5.5597 & 6.4678 & 0.825 & -24.762 & 13.642 \\
\hline & \multirow[t]{3}{*}{\begin{tabular}{|l|} 
Winter \\
\end{tabular}} & Summer & $-20.428^{*}$ & 6.4678 & 0.036 & -39.630 & -1.226 \\
\hline & & Autumn & -.1785 & 6.467 & 1.00 & -19.380 & 19.023 \\
\hline & & Spring & -5.7382 & 6.467 & 0.812 & -24.94 & 13.464 \\
\hline & \multirow[t]{3}{*}{ Spring } & Summer & -14.690 & 6.4678 & 0.160 & -33.892 & 4.512 \\
\hline & & Autumn & 5.5597 & 6.4678 & 0.825 & -13.642 & 24.762 \\
\hline & & Winter & 5.7382 & 6.467 & 0.812 & -13.464 & 24.940 \\
\hline
\end{tabular}


3.6.3 Relationship between concentrations of PAHs and two sampling points at each location

In order to investigate the effect of the two sampling points (inside and outside) at given location on the concentration of PAHs, using ANOVA- Multiple test. The results shows insignificant effects on the concentration of PAHs when transport sampling points between inside and outside at all chosen locations.

\subsubsection{Correlation Analysis}

The influence of meteorological parameters such as temperature, relative humidity and wind speed on the PAH emissions was analyzed by statistical package for social scientist (SPSS) program. Pearson correlation analysis was carried out to determine the correlation between TPAH and TSP with meteorological conditions at given location (and specified season). Tables (7-9) show the results of Pearson correlation for three sites in all seasons.

Table 7. Pearson Correlations between concentrations of TPAH and meteorological variables in all seasons at AL-Daura refinery.

Spring season

\begin{tabular}{|c|c|c|c|c|c|}
\hline parameters & $\begin{array}{c}\text { Temp. } \\
\left({ }^{\circ} \mathrm{C}\right)\end{array}$ & $\begin{array}{c}\text { Hum. } \\
\%\end{array}$ & $\begin{array}{l}\text { Wind } \\
(\mathrm{m} / \mathrm{s})\end{array}$ & $\begin{array}{c}\text { TSP } \\
(\mu \mathrm{g} \\
\left./ \mathrm{m}^{3}\right)\end{array}$ & $\begin{array}{l}\text { TPAHs } \\
\left(\mu \mathrm{g} / \mathrm{m}^{3}\right)\end{array}$ \\
\hline $\begin{array}{c}\text { TSP } \\
\left(\mu \mathrm{g} / \mathrm{m}^{3}\right)\end{array}$ & $\begin{array}{c}- \\
0.351\end{array}$ & 0.589 & 0.020 & 1 & \\
\hline $\begin{array}{l}\text { TPAHs } \\
\left(\mu \mathrm{g} / \mathrm{m}^{3}\right)\end{array}$ & $\begin{array}{c}- \\
0.261\end{array}$ & 0.822 & 0.420 & 0.897 & 1 \\
\hline
\end{tabular}

\section{Summer season}

\begin{tabular}{|c|c|c|c|c|c|}
\hline parameters & $\begin{array}{c}\text { Temp. } \\
\left({ }^{\circ} \mathrm{C}\right)\end{array}$ & $\begin{array}{c}\text { Hum } \\
\%\end{array}$ & $\begin{array}{l}\text { Wind } \\
(\mathrm{m} / \mathrm{s})\end{array}$ & $\begin{array}{c}\text { TSP } \\
(\mu \mathrm{g} \\
\left./ \mathrm{m}^{3}\right)\end{array}$ & $\begin{array}{l}\text { TPAHs } \\
\left(\mu \mathrm{g} / \mathrm{m}^{3}\right)\end{array}$ \\
\hline $\begin{array}{c}\text { TSP } \\
\left(\mu \mathrm{g} / \mathrm{m}^{3}\right)\end{array}$ & 0.671 & 0.491 & 0.614 & 1 & \\
\hline $\begin{array}{l}\text { TPAHs } \\
\left(\mu \mathrm{g} / \mathrm{m}^{3}\right)\end{array}$ & $\begin{array}{c}- \\
0.817\end{array}$ & $\begin{array}{c}- \\
0.379\end{array}$ & 0.468 & $0.966^{*}$ & 1 \\
\hline
\end{tabular}

Autumn season

\begin{tabular}{|c|c|c|c|c|c|}
\hline parameters & $\begin{array}{l}\text { Temp. } \\
\left({ }^{\circ} \mathrm{C}\right)\end{array}$ & $\begin{array}{c}\text { Hum. } \\
\%\end{array}$ & $\begin{array}{l}\text { Wind } \\
(\mathrm{m} / \mathrm{s})\end{array}$ & $\begin{array}{c}\text { TSP } \\
(\mu \mathrm{g} \\
\left./ \mathrm{m}^{3}\right) \\
\end{array}$ & $\begin{array}{c}\text { TPAHs } \\
(\mu \mathrm{g} \\
\left./ \mathrm{m}^{3}\right) \\
\end{array}$ \\
\hline $\begin{array}{c}\text { TSP } \\
\left(\mu \mathrm{g} / \mathrm{m}^{3}\right)\end{array}$ & 0.573 & 0.534 & $0.986^{*}$ & 1 & \\
\hline $\begin{array}{l}\text { TPAHs } \\
\left(\mu \mathrm{g} / \mathrm{m}^{3}\right)\end{array}$ & 0.027 & 0.072 & 0.845 & 0.800 & 1 \\
\hline
\end{tabular}

Winter season

\begin{tabular}{|c|c|c|c|c|c|}
\hline parameters & $\begin{array}{l}\text { Temp. } \\
\left({ }^{\circ} \mathrm{C}\right)\end{array}$ & Hum. & $\begin{array}{l}\text { Wind } \\
(\mathrm{m} / \mathrm{s})\end{array}$ & \begin{tabular}{|c|} 
TSP \\
$(\mu \mathrm{g}$ \\
$\left./ \mathrm{m}^{3}\right)$
\end{tabular} & $\begin{array}{c}\text { TPAHs } \\
(\mu \mathrm{g} \\
\left./ \mathrm{m}^{3}\right)\end{array}$ \\
\hline $\begin{array}{c}\text { TSP } \\
\left(\mu \mathrm{g} / \mathrm{m}^{3}\right)\end{array}$ & 0.222 & $\begin{array}{c}- \\
0.130\end{array}$ & $\begin{array}{c}- \\
0.743\end{array}$ & 1 & \\
\hline $\begin{array}{l}\text { TPAHs } \\
\left(\mu \mathrm{g} / \mathrm{m}^{3}\right)\end{array}$ & $\begin{array}{c}- \\
0.957^{*}\end{array}$ & $0.953^{*}$ & $\begin{array}{c}- \\
0.409\end{array}$ & $\begin{array}{c}- \\
0.261\end{array}$ & 1 \\
\hline
\end{tabular}

$* *$ correlation at $99 \%$ confidence level.

* correlation at $95 \%$ confidence level.

Table 8. Pearson Correlations between concentrations of TPAH and meteorological variables in all seasons at AL-Daura power plant.

Spring season

\begin{tabular}{|c|c|c|c|c|c|}
\hline parameters & $\begin{array}{c}\text { Temp. } \\
\left({ }^{\circ} \mathrm{C}\right)\end{array}$ & $\begin{array}{c}\text { Hum. } \\
\%\end{array}$ & $\begin{array}{c}\text { Wind } \\
(\mathrm{m} / \mathrm{s})\end{array}$ & $\begin{array}{c}\text { TSP } \\
\left(\mu \mathrm{g} / \mathrm{m}^{3}\right)\end{array}$ & $\begin{array}{c}\text { TPAHs } \\
\left(\mu \mathrm{g} / \mathrm{m}^{3}\right)\end{array}$ \\
\hline $\begin{array}{c}\mathrm{TSP} \\
\left(\mu \mathrm{g} / \mathrm{m}^{3}\right)\end{array}$ & $-.973^{*}$ & $.985^{*}$ & .931 & 1 & \\
\hline $\begin{array}{c}\mathrm{TPAHs} \\
\left(\mu \mathrm{g} / \mathrm{m}^{3}\right)\end{array}$ & -.946 & .883 & .684 & .893 & 1 \\
\hline
\end{tabular}


Summer season

\begin{tabular}{|c|c|c|c|c|c|}
\hline parameters & $\begin{array}{c}\text { Temp. } \\
\left({ }^{\circ} \mathrm{C}\right)\end{array}$ & $\begin{array}{c}\text { Hum. } \\
\%\end{array}$ & $\begin{array}{c}\text { Wind } \\
(\mathrm{m} / \mathrm{s})\end{array}$ & $\begin{array}{c}\text { TSP } \\
\left(\mu \mathrm{g} / \mathrm{m}^{3}\right)\end{array}$ & $\begin{array}{c}\text { TPAHs } \\
\left(\mu \mathrm{g} / \mathrm{m}^{3}\right)\end{array}$ \\
\hline $\begin{array}{c}\mathrm{TSP} \\
\left(\mu \mathrm{g} / \mathrm{m}^{3}\right)\end{array}$ & .457 & -.538 & -.838 & 1 & \\
\hline $\begin{array}{c}\mathrm{TPAHs} \\
\left(\mu \mathrm{g} / \mathrm{m}^{3}\right)\end{array}$ & $-.952^{*}$ & .942 & .822 & -.452 & 1 \\
\hline
\end{tabular}

Autumn season

\begin{tabular}{|c|c|c|c|c|c|}
\hline parameters & $\begin{array}{c}\text { Temp. } \\
\left({ }^{\circ} \mathrm{C}\right)\end{array}$ & $\begin{array}{c}\text { Hum. } \\
\%\end{array}$ & $\begin{array}{c}\text { Wind } \\
(\mathrm{m} / \mathrm{s})\end{array}$ & $\begin{array}{c}\text { TSP } \\
\left(\mu \mathrm{g} / \mathrm{m}^{3}\right)\end{array}$ & $\begin{array}{c}\text { TPAHs } \\
\left(\mu \mathrm{g} / \mathrm{m}^{3}\right)\end{array}$ \\
\hline $\begin{array}{c}\mathrm{TSP} \\
\left(\mu \mathrm{g} / \mathrm{m}^{3}\right)\end{array}$ & -.865 & .862 & -.846 & 1 & \\
\hline $\begin{array}{c}\mathrm{TPAHs} \\
\left(\mu \mathrm{g} / \mathrm{m}^{3}\right)\end{array}$ & -.631 & .823 & -.778 & .499 & 1 \\
\hline
\end{tabular}

Winter season

\begin{tabular}{|c|c|c|c|c|r|}
\hline parameters & $\begin{array}{c}\text { Temp. } \\
\left({ }^{\circ} \mathrm{C}\right)\end{array}$ & $\begin{array}{c}\text { Hum. } \\
\%\end{array}$ & $\begin{array}{c}\text { Wind } \\
(\mathrm{m} / \mathrm{s})\end{array}$ & $\begin{array}{c}\text { TSP } \\
\left(\mu \mathrm{g} / \mathrm{m}^{3}\right)\end{array}$ & $\begin{array}{c}\text { TPAHs } \\
\left(\mu \mathrm{g} / \mathrm{m}^{3}\right)\end{array}$ \\
\hline $\begin{array}{c}\text { TSP } \\
\left(\mu \mathrm{g} / \mathrm{m}^{3}\right)\end{array}$ & -.432 & .422 & -.662 & 1 & \\
\hline $\begin{array}{l}\mathrm{TPAHs} \\
\left(\mu \mathrm{g} / \mathrm{m}^{3}\right)\end{array}$ & .864 & -.799 & $.999^{* *}$ & -.629 & 1 \\
\hline
\end{tabular}

Table 9. Pearson Correlations between concentrations of TPAH and meteorological variables in all seasons at thermal power plant South of Baghdad.

Spring season

\begin{tabular}{|c|c|c|c|c|c|}
\hline parameters & $\begin{array}{c}\text { Temp. } \\
\left({ }^{\circ} \mathrm{C}\right)\end{array}$ & $\begin{array}{c}\text { Hum } \\
\%\end{array}$ & $\begin{array}{c}\text { Wind } \\
(\mathrm{m} / \mathrm{s})\end{array}$ & $\begin{array}{c}\text { TSP } \\
(\mu \mathrm{g} \\
\left.\left./ \mathrm{m}^{3}\right)\right)\end{array}$ & $\begin{array}{c}\text { TPAHs } \\
(\mu \mathrm{g} \\
\left./ \mathrm{m}^{3}\right)\end{array}$ \\
\hline $\begin{array}{c}\mathrm{TSP} \\
\left(\mu \mathrm{g} / \mathrm{m}^{3}\right)\end{array}$ & .786 & $-.978^{*}$ & -.314 & 1 & \\
\hline $\begin{array}{c}\mathrm{TPAHs} \\
\left(\mu \mathrm{g} / \mathrm{m}^{3}\right)\end{array}$ & -.009 & .044 & -.345 & - & 1 \\
\end{tabular}

Summer season

\begin{tabular}{|c|c|c|c|c|c|}
\hline parameters & $\begin{array}{c}\text { Temp. } \\
\left({ }^{\circ} \mathrm{C}\right)\end{array}$ & $\begin{array}{c}\text { Hum } \\
\%\end{array}$ & $\begin{array}{c}\text { Wind } \\
(\mathrm{m} / \mathrm{s})\end{array}$ & $\begin{array}{c}\text { TSP } \\
(\mu \mathrm{g} \\
\left.\left./ \mathrm{m}^{3}\right)\right)\end{array}$ & $\begin{array}{c}\text { TPAHs } \\
(\mu \mathrm{g} \\
\left./ \mathrm{m}^{3}\right)\end{array}$ \\
\hline $\begin{array}{c}\mathrm{TSP} \\
\left(\mu \mathrm{g} / \mathrm{m}^{3}\right)\end{array}$ & .079 & -.075 & -.044 & 1 & \\
\hline $\begin{array}{c}\text { TPAHs } \\
\left(\mu \mathrm{g} / \mathrm{m}^{3}\right)\end{array}$ & -.910 & .937 & .291 & -.419 & 1 \\
\hline
\end{tabular}

Autumn season

\begin{tabular}{|c|c|c|c|c|c|}
\hline parameters & $\begin{array}{c}\text { Temp. } \\
\left({ }^{\circ} \mathrm{C}\right)\end{array}$ & $\begin{array}{c}\text { Hum } \\
\%\end{array}$ & $\begin{array}{c}\text { Wind } \\
(\mathrm{m} / \mathrm{s})\end{array}$ & $\begin{array}{c}\text { TSP } \\
(\mu \mathrm{g} \\
\left.\left./ \mathrm{m}^{3}\right)\right)\end{array}$ & $\begin{array}{c}\text { TPAHs } \\
(\mu \mathrm{g} \\
\left./ \mathrm{m}^{3}\right)\end{array}$ \\
\hline $\begin{array}{c}\mathrm{TSP} \\
\left(\mu \mathrm{g} / \mathrm{m}^{3}\right)\end{array}$ & -.881 & .843 & -.340 & 1 & \\
\hline $\begin{array}{c}\text { TPAHs } \\
\left(\mu \mathrm{g} / \mathrm{m}^{3}\right)\end{array}$ & $-.955^{*}$ & .924 & -.280 & $.978^{*}$ & 1 \\
\hline
\end{tabular}

Winter season

\begin{tabular}{|c|c|c|c|c|c|}
\hline parameters & $\begin{array}{c}\text { Temp. } \\
\left({ }^{\circ} \mathrm{C}\right)\end{array}$ & $\begin{array}{c}\text { Hum } \\
\%\end{array}$ & $\begin{array}{c}\text { Wind } \\
(\mathrm{m} / \mathrm{s})\end{array}$ & $\begin{array}{c}\text { TSP } \\
(\mu \mathrm{g} \\
\left.\left./ \mathrm{m}^{3}\right)\right)\end{array}$ & $\begin{array}{c}\text { TPAHs } \\
(\mu \mathrm{g} \\
\left./ \mathrm{m}^{3}\right)\end{array}$ \\
\hline $\begin{array}{c}\mathrm{TSP} \\
\left(\mu \mathrm{g} / \mathrm{m}^{3}\right)\end{array}$ & .358 & -.297 & -.397 & 1 & \\
\hline $\begin{array}{c}\mathrm{TPAHs} \\
\left(\mu \mathrm{g} / \mathrm{m}^{3}\right)\end{array}$ & -.276 & .278 & -.704 & .675 & 1 \\
\hline
\end{tabular}

At AL-Daura refinery, the high significant positive correlation ( $\mathrm{r}=0.95,0.822$ at $95 \%$ confidence) between the total PAH concentration and the relative humidity was observed in the seasons winter and spring respectively as listed in the table (7). This could be due to the depositional effect on the particulate matter of PAHs in the gas phase as a consequence of environmental humidity [23].

The high significant negative correlation $(r=-0.957,-0.817)$ between the temperature and the total PAH concentration could be observed in both winter and summer while insignificant negative correlation in the spring and autumn 
seasons. There are high significant level and positive correlation values between TSP and TPAH (0.897, 0.966, 0.800) respectively in seasons spring, summer and autumn respectively, due to the higher concentration of the total suspended particulate which leads to the adsorption of more molecules of PAHs compounds [24]. For the wind rate studied, no statistical significance was obtained.

Table (8) illustrates high significant positive correlation $(\mathrm{r}=0.883, \quad 0942, \quad 0.823$ at $95 \%$ confidence) between the total PAH concentration and the relative humidity at AL-Daura power plant in spring, summer and autumn season respectively. There are high significant negative correlation was obtained between TPAH and temperature in spring, summer and autumn, while in winter high significant positive correlation. Relation between TPAH and TSP gave high significant positive correlation (0.893) in spring only, which might be attributed to moderate temperature, humidity and wind. At AL-Daura power plant wind rate study gave high significant positive correlation in spring, summer and winter seasons except autumn with negative correlation.

At thermal power plant South of Baghdad show high significant negative correlation (table 9) between TPAH and temperature in summer and autumn $(-0.91,-0.95)$ respectively, these result indicate that higher temperatures are associated with lower PAH concentrations. High significant negative correlation appears between TPAH and humidity $(0.924,0.937)$ in summer and autumn seasons respectively. Relation between TSP and TPAH gave high significant positive correlation
$(0.978,0.675)$ in autumn and winter seasons respectively, that might be attributed to the increase of TSP leading to more adsorption of TPAH.

\subsubsection{T-test}

To evaluate whether the concentrations of individual PAHs exceed the international permissible limits of compounds in the atmosphere air, data were treated using t-test. The results were listed in tables (13-15). 
Table 13. t-test results of comparison between concentrations of PAHs and international permissible limits at AL-Daura refinery.

\begin{tabular}{|c|c|c|c|c|c|c|c|}
\hline Compound & $\mathbf{N}^{*}$ & $\begin{array}{c}\text { international } \\
\text { permissible limits } \\
\left(\boldsymbol{\mu g} / \mathbf{m}^{\mathbf{3}}\right)\end{array}$ & Mean & $\begin{array}{c}\text { Std. } \\
\text { Error }\end{array}$ & Sig. & \multicolumn{2}{|c|}{$\begin{array}{c}\text { 95\% Confidence } \\
\text { Interval of the } \\
\text { Difference }\end{array}$} \\
\cline { 5 - 8 } & & & & & & Lower & Upper \\
\hline Fluoranthene & 16 & 0.00087 & 2.672 & 0.385 & 0.000 & 0.980 & 2.625 \\
\hline Benzo[a]anthracene & 16 & 0.00012 & 0.307 & 0.102 & 0.088 & -0.0311 & 0.405 \\
\hline Chrysene & 16 & 0.0087 & 0.373 & 0.086 & 0.005 & 0.102 & 0.470 \\
\hline
\end{tabular}

* Number of atmosphere air samples.

Table 14. t-test results of comparison between concentrations of PAHs and international permissible limits at AL-Daura power plant.

\begin{tabular}{|c|c|c|c|c|c|c|c|}
\hline Compound & $\mathbf{N}$ & $\begin{array}{c}\text { international } \\
\text { permissible } \\
\text { limits }\left(\boldsymbol{\mu g} / \mathbf{m}^{\mathbf{3}}\right)\end{array}$ & Mean & $\begin{array}{c}\text { Std. } \\
\text { Error }\end{array}$ & Sig. & \multicolumn{2}{|c|}{$\begin{array}{c}\text { 95\% Confidence } \\
\text { Interval of the } \\
\text { Difference }\end{array}$} \\
\cline { 4 - 8 } & & & & & \multicolumn{2}{|c|}{ Lower } & Upper \\
\hline Fluoranthene & 16 & 0.00087 & 6.21 & 2.47 & 0.048 & 0.060 & 10.615 \\
\hline Benzo[a]anthracene & 16 & 0.00012 & 1.27 & 0.51 & 0.041 & 0.052 & 2.249 \\
\hline Chrysene & 16 & 0.0087 & 1.75 & 0.74 & 0.040 & 0.083 & 3.238 \\
\hline
\end{tabular}

Table 15. t-test results of comparison between concentrations of PAHs and international permissible limits at thermal power plant South of Baghdad.

\begin{tabular}{|c|c|c|c|c|c|c|c|}
\hline Compound & $\mathbf{N}$ & $\begin{array}{c}\text { international } \\
\text { permissible } \\
\text { limits }\left(\boldsymbol{\mu} \mathbf{g} / \mathbf{m}^{\mathbf{3}}\right)\end{array}$ & Mean & $\begin{array}{c}\text { Std. } \\
\text { Error }\end{array}$ & sig. & \multicolumn{2}{|c|}{$\begin{array}{c}\mathbf{9 5 \%} \text { Confidence } \\
\text { Interval of the } \\
\text { Difference }\end{array}$} \\
\cline { 4 - 8 } & & & & & Lower & Upper \\
\hline Fluoranthene & 16 & 0.00087 & 11.26 & 4.43 & 0.033 & 0.941 & 19.848 \\
\hline Benz[a]anthracene & 16 & 0.00012 & 1.60 & 0.55 & 0.016 & 0.319 & 2.646 \\
\hline Chrysene & 16 & 0.0087 & 2.63 & 1.28 & .066 & -0.190 & 5.270 \\
\hline
\end{tabular}

Fluoranthene, Benzo[a]anthracene and Chrysene compounds show statistically significant ( $\mathrm{p}<0.05$ ), while the other PAHs compounds do not show significant differences ( $p>0.05$ at $95 \%$ confidence) between concentrations and International permissible limits. From results illustrated high contamination in the study area and surrounding by many PHAs especially Fluoranthene, Benzo[a]anthracene and Chrysene.

\section{Acknowledgments}

The authors wish to thank Prof Dr. Christoph Schüth, vice -Dean, department of Materials and Geosciences , Darmstadt university for permission to use GC/MS divece. The authors are very grateful to Dr. Tom Schiedek and Ms 
Stefanie Schmidt, department of Materials and Geosciences, Darmstadt university, for cooperation in the analysis of samples.

\section{References}

1- Holland, W. W., Bennett, A. E., Cameron, I. R., Florey, C. V., Leeder, S. R., Shilling, R. S. F.,Swan, A. V. \& Waller, R. E. 1979, Health Effects of Particulate Pollution: Reappraising the Evidence. Am Journal Epidemiol, 110(5), pp. 525- 659.

2- Martı'nez, E., Gros, M., Lacorte S. and Barcelo', D. 2004, J. Chromatogr. A 1047 181.

3- Wiliams, P.T. and Besler, S. 1996, J. Anal. Appl. Pyrol. 30, 351.

4- Lee, H. Mi, Lin, W., Wu, T., Wang, T. and Chao, L. H. 1992, J. Environ., 290.

5- Yang, H. Lee, W. Chen, S. and Lai, S. 1998, J. Hazard. Mater. $60 \mathrm{Fd}$. Chem. 159.

6- Charles, D.T., Ide, F., McLachlan, J.A. and Arnold, S.F. 1996, Biochem. Biophys. Res. Commun. 229, 102.

7- Kizu, R., Ishii, K. and Kobayashi, J. 2000, Mater. Sci. Eng. C 12, 97.

8- Becher, G., Haugen, A. and Bjorseth, A. 1984, Carcinogenesis 5, 1-647.

9- Buckley, T.J. and Lioy, P.J. 1992, Br. J. Ind. Med. 49113.

10- Howsam, M., Jones, KC. 1998, Sources of PAHs in the environment. In: Neilson AH(ed). Anthropogenic compounds. PAHs and related compounds. Springer, Berlin, Germany, 137-174.

11- Blumer, M. 2003, Polycyclic aromatic compounds in nature., Scientific American 35-45.

12- Schmidt, S. N., Smith, K. E,. Holmstrup, M. and Mayer, P. 2013, Environmental
Toxicology and Chemistry, 32(2), 361369.

13-Daly, A. and Zannetti, P. 2007, "An introduction to air pollution - definitions, classifications, and history". Published by The Arab School for Science and Technology (ASST). Ch.1.

14- Tabatabaei, F. 2012, Health Scope. 1(3), 99-100.

15- Krishnakumar, V. 1994, Encology., 9 (4), 6-9.

16- Nordell, B., Global and Planetary Change. 38, 305-312, 2003.

17- Israa M. H. Almousawi., 2014, "Environmental Assessment of Some Polycyclic Aromatic Hydrocarbons Concentrations in the Atmospheric Air at Certain Areas of Baghdad City", Ph.D. Thesis. Department of Chemistry, College of Science - University of Baghdad, Baghdad, Iraq.

18-Afaj, A. H., Shanshal, M., , Almousawi I. M. H. and Safauldeen, 2014, A. A., IJCPE Vol.15 No.3.

19- Almousawi I. M. H., Shanshal, M. and Afaj, A. H. 2015, Iraqi Journal of Biotechnology, 14 (2), 403-414.

20-Brebbia, C. A., Longhurst, J.W.S. and Popov V. 2011, " Air Pollution XIX", Conference, WIT Press, UK.

21- Delgado-Saborit, J. M., Aquilina, N., Baker, S., and Harrison, R. M. 2010, Anal. Methods, 2, 231-242.

22- Mitsova, D. Vos, J. Gardinali, P. and Stafeychuk, I. 2011, J. of Environmental Protection, 2, 1317-1330.

23-Lee, B-K. and Lee, C-H. 2008, Environ. Monit. Assess, 136, 21-33.

24- Alias, M., Hamzah, Z. and Kenn, L. S. 2007, The Malaysian J. of Anal. Sci., 11(1), 255-2 


\section{التحليل الإحصائي لتراكيز الهيدروكربونات الأروماتية متعددة العلقات في مدينة بغداد}

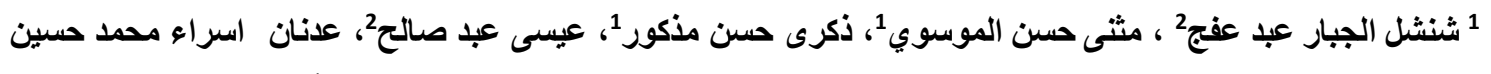

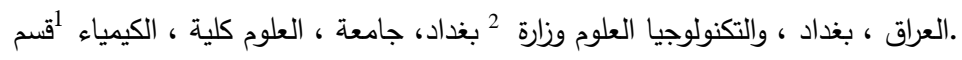

في عينات الهواء الجوي والتي تم جمعها في الفترة من (PAHs) قيست تراكيز 16 مركب هيدروكربوني عطري متعدد الحلقات أبريل 2012 إلى مارس 2013، في ثلاث مواقع لمدينة بغداد ( مصفى الدورة ومحطة الدورة الكهبائية ومحطة كهرباءجنوب بغداد الحرارية). عينات الهواء الجوي استخلصت باستخدام جهاز السوكسليت.

متوسط تراكيز قيس PAH من أجل تعيين مركبات GC-MS بعد الالاستخلاص، تم تحليل 138 عينة باستخدام جهاز الفردية في الهواء المحيط لمختلف الفصول. ودلت النتائج إلى انتشار معظم الهيدروكربونات العطريه متعددة الحلقات PAH PAH في ثلاث مواقع لمدينة بغداد. تم دراسة تاثير كل من مواقع اخذ العينات والمواسم والظروف الجوية على تراكيز PAHs دلت النتائج على وجود علاقة إيجابية بين درجة الحرارة وتراكيز المركبات الهيدروكربونية العطريه باستخدام برنامج التركيز الكلي العطرية للهيدروبونات المختارة المواقع 